\title{
Surgical role in the management of peritoneal dialysis catheters - evidence and proposals to improve current practices
}

\author{
Rita Vicente ${ }^{1}$ (iD), Rui Silva ${ }^{1}$ (iD), Ricardo Santos ${ }^{1}$ (iD), Anabela Rodrigues ${ }^{2}$ (iD), Manuel Amoedo ${ }^{1}$ (iD \\ ${ }^{1}$ Nephrology Department, Hospital Espírito Santo de Évora, Portugal \\ 2 Nephrology Department, Centro Hospitalar Universitário do Porto, Portugal
}

\section{ABSTRACT}

Peritoneal dialysis (PD) relies on adequate function of PD catheter. Mechanical complications are a major cause of dropout among PD patients. A prompt timing and an appropriate intervention may overcome these problems with catheter salvage, without interrupting PD treatment. Many Portuguese centers still lack a proper articulation between nephrology and surgical departments. We describe current available surgical approaches in the implantation of PD catheters, management of mechanical complications, exit-site infections and other abdominal surgical procedures. Our aim is to reinforce the need for an established protocol of action between these departments as occurs with vascular access in hemodialysis.

Key-words: peritoneal dialysis; peritoneal dialysis catheters, mechanical complications

(C) 2021 Portuguese Journal of Nephrology \& Hypertension. Published by Publicações Ciência \& Vida This is an open access article under the CC BY-NC-ND license (http://creativecommons.org/licenses/by-nc-nd/4.0/).

\section{INTRODUCTION}

New evidence regarding catheter implantation and management has emerged in recent years, which can ameliorate the existing practices, potentially improving peritoneal dialysis (PD) survival.

Mechanical complications are one of the main reasons of dropout among PD patients. In Portugal they are the third cause of PD withdrawal. In 2018 and 2019, an increase in dropout rate for mechanical problems was reported: $17.5 \%$ and $15.2 \%$ respectively compared to 9.4-11.3\% in the years between 2014-2017 (data from the Portuguese Registry of Dialysis and Transplantation $)^{1}$.

Mechanical complications appear to have an increased incidence in the earliest period in PD (first 3 months) with a significant decrease after 1-year, as described by Kolesnyk et $a l^{2}$. This notion reflects the importance of the practices in pre, peri and postoperative periods by a multidisciplinary PD access team. Therefore, the moment of patient evaluation, with catheter selection, determination of insertion and exit-site location, and the selection of placement procedure accordingly to the patient's history and characteristics should have a dedicated time for an optimal assessment.

Another factor associated with improvement of perioperative adverse effects is a center with great number of catheter implantations, which can lead to a better training and accumulated experience and skills ${ }^{3}$.

A prompt timing and an appropriate intervention can overcome possible mechanical and infectious problems with catheter salvage, without interrupting PD treatment. Many centers still lack a proper articulation between nephrology and surgical departments, leading to a delay in the resolution of these complications with a consequent temporary transition to hemodialysis (HD), frequently by a central venous catheter. An inadequacy in the timing of response can also be discouraging to PD patients and precipitate a definitive transference to HD.

In this article, our aim is to show the current available surgical approaches in the implantation of PD catheters, management of mechanical complications, exit-site infections and other abdominal surgical procedures and to reinforce the importance of an established articulation amongst nephrologists and surgeons with institutional pathways.

\section{CATHETER PLACEMENT PROCEDURES}

A PD catheter can be inserted by blind techniques - percutaneous needle-guidance technique with or without image guidance (fluoroscopy and/or ultrasonography), peritoneoscopy assisted technique (Y-TEC) or by direct visualization techniques - open surgical dissection, basic and advanced laparoscopy. Advanced laparoscopy adds procedures and skills to overcome the risk of peritoneal catheter disfunction in more complex patients.

A 2018 meta-analysis that compared, for the first-time, advanced laparoscopy, basic laparoscopy and open dissection showed significant superiority in advanced laparoscopy over the other procedures ${ }^{4}$. Previous studies did not report significant differences between the various techniques in terms of negative outcomes and catheter survival because patients submitted to basic and advanced were grouped together. This was most likely the flaw that led to inconsistent results ${ }^{5-8}$. 
In fact, in advanced laparoscopy, there was a considerable reduction in negative outcomes: less catheter obstruction, catheter migration, pericannular leak and incisional hernias, and a better catheter survival at 1 and 2-years. This may be explained by the preventive measures taken during the procedure: rectus sheath tunneling - that allows a longer path in the sheath of the rectus muscle, preventing catheter migration, omental wrapping, pericatheter hernias and leaks; selective omentopexy - which consists of omental fixation to the anterior abdominal wall of the upper abdomen in the case of a redundant omentum; adhesiolysis in the presence of previous adhesions, preventing intraperitoneal compartmentalization with incomplete drainage; correction of occult hernias not identified by clinical evaluation, and resection of intraperitoneal structures that could interfere with the catheter (e.g. epiploic appendices of the sigmoid colon) ${ }^{9}$.

The 2019 update in the International Society for Peritoneal Dialysis (ISPD) guidelines on the peritoneal dialysis access ${ }^{10}$ recommended advanced laparoscopy as the better placement procedure for minimizing the risk of mechanical complications (ISPD level of evidence: 1B). Nevertheless, they maintain the suggestion that the choice of technique should be based on center possibilities, operator expertise and patient characteristics (ISPD level of evidence: not graded). The preference for advanced laparoscopy (first option) and open surgical dissection is also suggested over the other techniques when there is a positive history of previous major surgery or peritonitis.

All insertion methods allow the embedding technique, also known as Moncrief-Popovich, which facilitates a programmed implantation of PD catheters, as occurs with the vascular access for HD. In addition to the numerous potential advantages of catheter embedding (better healing process, needless of break-in period, reduced catheter induced peritonitis, greater patient acceptance) it can also improve the implantation schedule ${ }^{11}$. However, this technique should not be performed if there is a risk of blood accumulation in the peritoneal cavity, when adhesiolysis, omentopexy or hernia correction is needed to enable optimal catheter placement by advanced laparoscopy ${ }^{10}$. In these situations, peritoneal dialysis catheter should be externalised to permit a flushing protocol, reducing the risk of intraluminal clots and adhesion formation ${ }^{10}$.

Another aspect to mention is the necessity of having a PD team with experience with the different types of catheters and the diverse possibilities of exit-site locations. This expertise is particularly difficult in small PD centers, but organizational steps should be taken in order to promote protocols of cooperation with reference centers of peritoneal access management.

Obesity is a worldwide problem and it is important to obtain an easily accessible exit-site location especially in this population of PD patients in order to minimize exit-site and tunnel infections and the nuisance to the patient. To overcome this issue, two-piece extended catheters were created to permit a presternal exit site or other remote locations in upper abdomen and back regions ${ }^{12}$. Other potential candidates to these catheters are patients with stomas, floppy abdominal skin folds, fecal and urinary incontinence, suprapubic catheters and behavior problems ${ }^{12}$. These catheters are composed of one-cuff abdomen catheter joined with a two-cuff extension segment through a titanium connector and are tunneled to the remote location with a tunneling $\operatorname{rod}^{11,13}$. They can be placed by any of the previously described techniques. Although two-piece extended catheter utilization was introduced a long time ago (in 1992, by Twardowski ${ }^{13}$ ), there is little experience in Portugal. Thus, there is room for improvement in this area.

Additionally, although advanced laparoscopy has proven superior to the other techniques, a proper patient selection for each procedure, taking into account patient history (previous interventions, peritonitis, biotype, tolerance for general anesthesia), center capacity and expertise is of utmost importance. Only by combining these factors can we achieve optimal results.

It must also be taken into account that catheter insertion by nephrologists seems to improve PD incidence, allowing PD catheters to be implanted in time ${ }^{14,15}$. This avoids long waiting lists induced by tight operating theater schedules and anesthetist's availability which may discourage potential PD patients and promote dialysis initiation by HD. Therefore, we believe that in the absence of history of abdominopelvic surgeries, abdominal wall hernias or significant obesity, catheter placement by nephrologists should be the first choice of catheter implantation.

Ambulatory surgical procedures have been put into practice and should be more broadly recommended, avoiding the burden of hospital admissions and, therefore, allowing a more cost-efficient intervention, with greater convenience and comfort to patients in the field of peritoneal access implantation, but the majority of hospitals have not promoted this access circuit yet.

In more complex patients, catheter implantation may not be feasible as an outpatient procedure, particularly if immediate induction of intermittent automated peritoneal dialysis is needed. However domiciliary hospitalization for such technical causes might be an organisational resource.

Furthermore, clinicians still lack a "dialysis access corridor" for surgical peritoneal catheter complications that need urgent treatment. That can be achieved by raising hospital administration awareness to this particular issue and by establishing a close articulation with the surgical department of each hospital. If it is verified that this cannot be accomplished, the creation of a peritoneal access center of reference, at a regional level, could be a possibility. This, as previously mentioned, is a measure that can potentially prevent a temporary transition to HD and avoid CVC placement, preserving vascular integrity, and even assure patients' confidence in PD as a worthwhile renal replacement technique.

\section{MECHANICAL COMPLICATIONS}

\section{Catheter dysfunction}

Catheter dysfunction is a frequent complication in PD and presents with outflow or inflow-outflow problems. The most common and easily reversible cause of outflow dysfunction is constipation. Other 
conditions which frequently lead to one-way dysfunction include bladder distention, catheter migration, extrinsic tissue attachment (omental entrapment, adhesions, uterine tube, epiploic appendices of colon), and compartmentalizing adhesions. Catheter obstruction due to fibrin or blood clots and kinking of the catheter are associated with two-way dysfunction.

The diagnostic and therapy approach should progress from conservative and less invasive to more aggressive interventions (ISPD level of evidence: not graded) ${ }^{10}$.

A possible model of catheter dysfunction's management is represented in Figure 1.

\section{Laparoscopy}

Laparoscopy is currently the gold standard for catheter malfunction treatment should the first measures referred in Figure 1 prove unsuccessful. This technique enables direct visualization of malfunction etiology, allowing the resolution of the problem. Laparoscopy is associated with a higher length of catheter patency with a reported long-term clinical success of $62.5-100 \%{ }^{16-19}$.

It allows patients to immediately restart treatment, using an intermittent dialysis protocol, in supine position, with low volume to prevent abdominal leaks and hernias.

Laparoscopy addresses to the primary cause through many available procedures: omentopexy, adhesiolysis, catheter redirection to a pelvic location, laparoscopic milking of occluded catheters, epiplopectomy, salpingectomy, colopexy $\mathrm{y}^{20}$.

In the case of catheter tip migration, this technique also enables a suture sling around the catheter in the lower abdomen to prevent migration recurrence which will not hamper catheter removal in the future ${ }^{20,21}$. Catheter tip suture to pelvic structures is not advised for

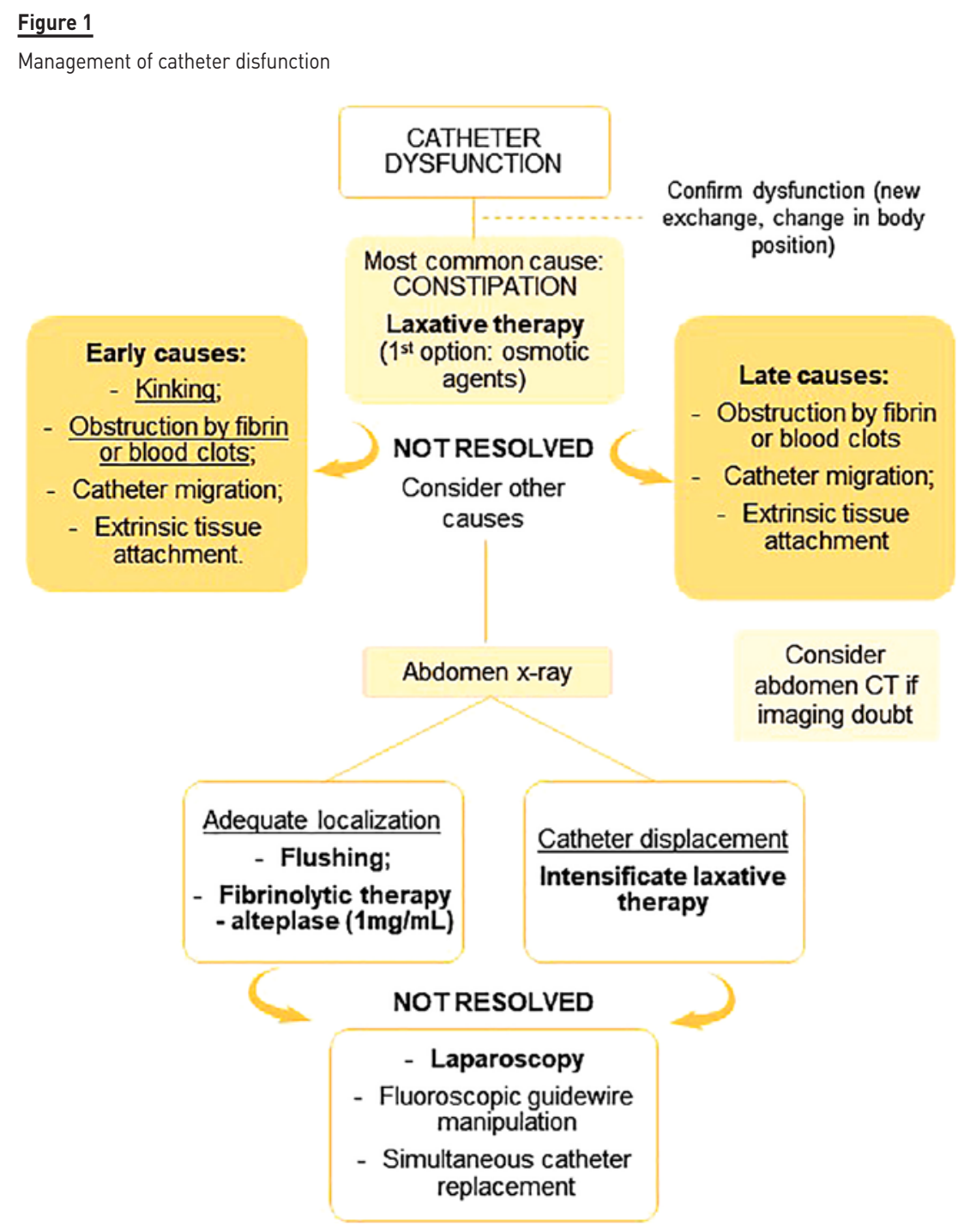


the likelihood of suture erosion and it may make catheter removal when necessary difficult ${ }^{21}$.

To minimize risk of abdominal leaks a watertight suture closure of all laparoscopic port sites is important.

Laparoscopy has some disadvantages such as requiring general anesthesia and an available operating theater, longer duration of the intervention and higher costs than the other techniques.

In essence, restoring catheter function is of utmost importance to prevent temporary transition to hemodialysis. Therefore, a protocol of urgent intervention must be established in these situations between the nephology and surgery departments.

\section{Fluoroscopic guidewire manipulation}

Radiological intervention is a minimally invasive approach with a low complication rate but with a reported success rate of $25-67 \%$. Therefore, it frequently requires multiple interventions, potentially discouraging patients from this PD modality $22-26$.

Guidewire manipulation of swan-neck catheters may be challenging owing to their arc bend shape and could lead to tunnel trauma. ${ }^{21}$

An analysis of 70 non-functional catheters treated with fluoroscopic manipulation identified two predictors of successful procedure: pelvic location of the catheter tip versus upper abdominal location (73.5 versus $42.9 \%$ ) and catheters that were initially functional compared with those that failed at exteriorization ( $75 \%$ versus $46.7 \%)^{22}$.

The association between the timing of dysfunction after implantation are described in other reports ${ }^{23,26-27}$. Hevia C. ${ }^{27}$ reported a percentage of $84 \%$ unsuccessful procedures in the first 3 months of catheter life.

A strong association between fluoroscopic manipulation failure and previous history of abdominopelvic surgery and peritonitis is also described, implying that the presence of adhesions may be the cause of these outcomes 21,24 .

These findings may potentially help us select which patients could have a greater chance of sucess with this technique for a better resource optimization. According to these results, a patient with a catheter tip not far from pelvic location, with a catheter that had some functionality before the disfunction and without a history of abdominopelvic surgery or peritonitis, could be a more suitable candidate for this technique. Beyond that, this technique is a possible option for patients that cannot go under general anesthesia.

The fluoroscopic guidewire manipulation of peritoneal catheter is a technique rarely performed in Portugal. It could be a way to a faster response to catheter dysfunction resolution in selected patients, despite the lower successful rates comparing to the laparoscopic approach. This might be possible using the available angiography suites already utilized in vascular access management.

\section{Simultaneous catheter replacement by open surgical technique}

This procedure doesn't allow the identification of what is causing the malfunction. It's associated with the high probability of recurrence. In one study only a $37.5 \%$ success rate was demonstrated ${ }^{16}$.

Furthermore, after catheter replacement a resting period is recommended in order to facilitate wound healing to prevent abdominal and pericatheter leaks and hernias. In patients without adequate residual renal function (RRF), it frequently involves transitory transition to hemodialysis.

This technique should be reserved for when laparoscopy is not possible, in the case of patient intolerance to general anesthesia or for logistic reasons.

\section{Abdominal wall hernias}

PD patients have a higher probability of hernia occurrence than the general population, possibly caused by increased intra-abdominal pressure (IAP) and deteriorated connective tissue ${ }^{28}$. These factors also predispose to high recurrence rates ${ }^{29}$.

Hernias in PD patients should be repaired as early as possible to prevent complications and emergency surgery in the case of incarceration or strangulation ${ }^{30}$.

Hernia correction in these patients must allow PD resumption in the postoperative period while at the same time minimizing the potential risk of abdominal leaks. Many authors recommend that hernias in $\mathrm{PD}$ patients should be repaired using prosthetic material, even the smaller ones ${ }^{31}$. Herniorrhaphy should be performed with tension-free techniques ${ }^{31}$. As described by Ureña et al the hernial sac must be dissected and inverted without opening it, both in midline as in inguinal hernias $^{28}$. If the sac is opened, a watertight closure should be made with an absorbable suture ${ }^{28}$.

After the surgery, IAP should be maintained as low as possible to reduce the risk of complications, by using low volumes, with the patient in supine position, with dry-day and if possible, by automated peritoneal dialysis ${ }^{32}$. The timing of PD restart depends on patient's RRF. Patients with acceptable RRF may withstand discontinuation of treatment for a week, whereas those with low or without RFF must resume $\mathrm{PD}$ after hernia repair or be transferred temporarily to hemodialysis for a few weeks ${ }^{21}$.

\section{Surgical catheter salvage in infectious complications}

Exit-site infection (ESI) of the peritoneal dialysis catheter remains a significant cause of catheter loss. ESIs are considered chronic when they reappear after a 2-4 week course of adequate antibiotic therapy and are caused by the same agent ${ }^{33}$. The major risk of chronic ESI is the development of a catheter-associated peritonitis, if the infecting organisms track along the catheter into the peritoneal cavity. To prevent this complication and consequent catheter removal, many techniques have been developed to eradicate the probable site of bacterial 
seeding, the superficial cuff, and/or relocate the exit-site to a non-colonized area.

According to the ISPD 2019 guidelines ${ }^{10}$, ultrasonography can be useful in procedure selection. If fluid is not detected around the external cuff and infection is caused by poor exit-site, the splicing technique may resolve chronic ESI. On the other hand, if fluid is detected around the external cuff, its shaving with cleansing of the affected tissue can effectively manage the infectious process.

As described below, there are several techniques to address this problem. In the literature, many distinct ways to perform each technique are illustrated, some even combining them, such as Fukasawa ${ }^{34}$ that combined shaving procedure with catheter splicing.

In this article we mention only the fundamentals of each procedure.

\section{Catheter splicing}

Catheter splicing consists of replacement of the external infected part of the catheter by a new segment, joined by a titanium connector. As mentioned before, this technique is favored when a better exit-site location is needed to improve the exit-site care, minimizing the risk of new infections (e.g. to the upper abdomen and chest locations) $)^{31}$. The procedure can be performed with local or general anesthesia, depending on the tunneling extension. It is made with an incision close to or in the previous insertion site to expose the intercuff segment ${ }^{35,36}$. The catheter is split up at this level, conserving a 1.5- to 2-cm segment in the deep-cuff side ${ }^{36}$. The extension is attached to the stump by a titanium connector ${ }^{35,36}$. Both catheter ends are tied with prolene stitches to each other ${ }^{35,36}$. The external segment is tunneled to the new exit-site, the wound is closed, and the previous catheter is removed ${ }^{36}$.

\section{Unroofing of the tunnel track and external cuff shaving}

This procedure consists of making an elliptical incision around the exit-site after infiltration of the area with local anesthetic. The incision is extended to the superficial cuff location. The exit-site skin and inflammatory tissue is removed. Then the external cuff is stripped. Many describe the use of a scalpel, but in our experience, we recommend the use of a blunt instrument, such as a Kelly curved forceps, to prevent catheter puncture. The catheter is immobilized in the medial corner of the incision with sterile adhesive strips for better exit-site cicatrisation, with the previous external cuff area exteriorized. The incision heals by secondary intention ${ }^{37}$.

A variation to this technique is the en bloc resection, where the exit-site skin to the superficial cuff location is removed in a teardrop shape, to minimize the exposure of the tissue to the infected material. The inflammatory tissue is removed with the help of an electrosurgical knife. Cuff stripping is made as previously described. The other difference is that the wound is sutured by an absorbent monofilament thread ${ }^{38}$.

\section{Exit-site relocation with external cuff shaving}

Initially, the catheter is soaked with chlorhexidine $2 \%$ solution for at least 5 minutes. This procedure is made under local anesthesia. An incision close to the superficial cuff is made and after the exposition of the external cuff, it is stripped off, using forceps. After the soaking period, the catheter is retracted through the cuff incision site and then re-soaked in chlorhexidine for another 5 minutes. After that, a new exit-site location is created, with the help of a tunneling too ${ }^{39}$.

This procedure may potentially be beneficial in patients with a deep and/or distant from the exit-site external cuff. Being minimally invasive, it reduces the risk of hemorrhage and morbidity of a large incision healing by secondary intention.

In all techniques maintaining oral antibiotic therapy for a further 2 to 4 weeks after surgery is recommended $31,37,39$.

\section{Other abdominal surgical procedures}

Laparoscopic approach has become a preferential option with PD patients in need of abdominal surgery ${ }^{40}$. It's a less invasive procedure than open surgery, which leads to lesser abdominal adhesion formation and better preservation of peritoneum integrity ${ }^{41}$. It involves smaller incisions, which reduces the risk of complications when PD is resumed, such as abdominal leakage ${ }^{42}$ and hernia formation.

Many case series of laparoscopic cholecystectomies, appendicectomies, nephrectomies, colectomies, and bariatric surgeries have been published. A review of the literature of laparoscopic surgery in PD patients published in $2017^{40}$ concluded that this technique permits an earlier resumption of PD treatment. Although in most cases it meant a transitory transition to hemodialysis, many case reports describe early PD resumption safety in laparoscopic cholecystectomy. Some aspects may be important for the prevention of postoperative complications. An adequate suturing of peritoneum, fascia, and abdominal wall providing a correct seal of the port sites can minimize complication risk ${ }^{43}$. Another important aspect is PD prescription after surgery, which must consider lowering intraabdominal pressure as much as possible, as has been previously described in the management of abdominal hernias ${ }^{32}$.

Laparoscopic cholecystectomy may also be performed at the time of catheter placement, in patients with symptomatic biliary tract disease, as long as they do not have active infection ${ }^{10,44}$. This measure can avoid gallstone-related complications, such as acute cholecystitis, that can interfere with PD survival ${ }^{44}$.

\section{CONCLUSIONS}

We feel there is still a lack of urgency in the resolution of PD catheter-associated complications, with a transient transference to HD generally well accepted, even among nephrologists. However, the prompt salvage of PD catheter for mechanical or infectious complications has an important role in reducing patient morbidity, financial burden, and technique dropout. The surgical department plays a fundamental role in the management of PD catheter from implantation, treatment of catheter dysfunction, abdominal wall complications, and other abdominal interventions. Laparoscopic interventions can potentially deal with abdominal surgery, minimizing the transference to HD. 
An established articulation between the nephrology and surgical departments is of utmost importance to implement a successful PD program. The creation of a "dialysis access corridor" is needed for a better management of complex patients and emergent complications not amenable within ambulatory surgery schedules.

Disclosure of potential conflicts of interest: none declared.

\section{References}

1. Portuguese Registry of Dialysis and Transplantation. Available at https://www.spnefro.pt/tratamento_da_doenca_renal_terminal. Accessed May 20, 2021.

2. Kolesnyk et al. Time-dependent reasons for peritoneal dialysis technique failure and mortality. Peritoneal Dialysis International 30:170-7.

3. Yoshitaka Kinoshita et al. Hospital-volume effects on perioperative outcomes in peritoneal dialysis catheter implantation: analysis of 2,505 cases. Perit Dial Int. 2018 Nov-Dec;38(6):419-423.

4. Shrestha BM, Shrestha D, Kumar A, Shrestha A, Boyes SA, Wilkie ME. Advanced laparoscopic peritoneal dialysis catheter insertion: systematic review and meta-analysis. Perit Dial Int 2018; 38:163-71.

5. Qiao Q, Zhou L, Hu K, Xu D, Li L, Lu G. Laparoscopic versus traditional peritoneal dialysis catheter insertion: a meta-analysis. Ren Fail 2016; 38(5):838-48.

6. Chen $Y$, Shao $Y, X u$ J. The survival and complication rates of laparoscopic versus open catheter placement in peritoneal dialysis patients: a meta-analysis. Surg Laparosc Endosc Percutan Tech 2015; 25(5):440-3.

7. Hagen SM, Lafranca JA, Steyerberg EW, IJzermans JN, Dor FJ. Laparoscopic versus open peritoneal dialysis catheter insertion: a meta-analysis. PLOS One 2013; 8(2):e56351.

8. Xie H, Zhang W, Cheng J, He Q. Laparoscopic versus open catheter placement in peritoneal dialysis patients: a systematic review and meta-analysis. BMC Nephrol 2012; 13:69.

9. Crabtree JH. Selected best demonstrated practices in peritoneal dialysis access. Kidney Int Suppl 2006(103):S27-37.

10. Crabtree et al. ISPD Guidelines/Recommendations - Creating and Maintaining Optimal Peritoneal Dialysis Access in the Adult Patient: 2019 Update

11. John H. Crabtree, Kai-Ming Chow. Peritoneal Dialysis Catheter Insertion. Seminars in Nephrology. 2017.

12. Penner T, Crabtree JH. Peritoneal dialysis catheters with back exit sites. Perit Dial Int. 2013; 33:93-6

13. Twardowski ZJ, et al. Swan neck presternal ("bath tub") catheter for peritoneal dialysis. Adv PeritDial.1992; 8:316-24.

14. Goh BLGY, Chew SE, Dalimi MS. Does peritoneal dialysis catheter insertion by interventional nephrologists enhance peritoneal dialysis penetration? Semin Dial. 2008; 21(6):561-7.

15. Li PKCK. Importance of peritoneal dialysis catheter insertion by nephrologists: practice makes perfect. Nephrol Dial Transplant. 2009; 24(11):3274-6.

16. Alabi A, Dholakia S, Ablorsu E. The role of laparoscopic surgery in the management of a malfunctioning catheter. Ann R Coll Surg Engl 2014; 96:593-6.

17. Ovnat A, Dukhno O, Pinsk I, Peiser J, Levy I. The laparoscopic option in the management of peritoneal dialysis catheter revision. Surg Endosc 2002; 16:698-9.

18. Ogunc G. Malfunctioning peritoneal dialysis catheter and accompanying surgical pathology repaired by laparoscopic surgery. Peri Dial Int 2002; 22:454-62.

19. Salgaonkar $\mathrm{H}$, et al. Minimally invasive surgery for salvage of malfunctioning peritoneal dialysis catheters. J Minim Access Surg. 2019; 15(1):19-24.

20. Crabtree J. Development of surgical guidelines for laparoscopic peritoneal dialysis access: Down a long and winding road. Peri Dial Int 2015; 35:241-4.

21. Daurgidas J, Blake P, Ing T. Handbook of dialysis. Philadelphia, United States: Lippincott Williams and Wilkins; 2014

22. Miller $\mathrm{M}$, et al. Fluoroscopic manipulation of peritoneal dialysis catheters: outcomes and factors associated with successful manipulation. Clin J Am Soc Nephrol 2012; 7:795-800.

23. Savader SJ, Lund G, Scheel PJ, Prescott C, Feeley N, Singh H, et al. Guide wire directed manipulation of malfunctioning peritoneal dialysis catheters: a critical analysis. J Vasc Interv Radiol 1997; 8:957-63.

24. Dobrashian RD, Conway B, Hutchison A, Gokal R, Taylor PM. The repositioning of migrated Tenckhoff continuous ambulatory peritoneal dialysis catheters under fluoroscopic control. $\mathrm{Br} J$ Radiol $1999 ; 72: 452-6$.
25. Moss JS, Minda SA, Newman GE, Dunnick NR, Vernon WB, Schwab SJ. Malpositioned peritoneal dialysis catheters: a critical reappraisal of correction by stiff-wire manipulation. Am J Kidney Dis 1990; 15:305-8.

26. Degesys GE, Miller GA, Ford KK, Dunnick NR. Tenckhoff peritoneal dialysis catheters: the use of fluoroscopy in management. Radiology 1985; 154:819-20.

27. Covadonga Hevia et al. Alpha replacement method for a displaced peritoneal catheter: a simple and effective maneuver. Adv Perit Dial 2001; 17:138-41.

28. García-Ureña MA, Rodríguez CR, Ruiz VV, Hernández FJ, Fernández-Ruiz E, Gallego JM, García MV. Prevalence and management of hernias in peritoneal dialysis patients. Perit Dial Int 2006; 26(2):198-202

29. Wetherington GM, Leapman SB, Robison RJ, Filo RS. Abdominal wall and inguinal hernias in continuous ambulatory peritoneal dialysis patients. Am J Surg 1985; 150:357-60.

30. Gianetta E, Civalleri D, Serventi A, Floris F, Mariani F, Aloisi F, Saffioti S. Anterior tension-free repair under local anesthesia of abdominal wall hernias in continuous ambulatory peritoneal dialysis patients. Hernia 2004; 8:354-7.

31. John H. Crabtree. Rescue and salvage procedures for mechanical and infectious complications of peritoneal dialysis. The International Journal of Artificial Organs 2006; 29(1):67-84.

32. John H. Crabtree. Hernia repair without delay in initiating or continuing peritoneal dialysis. Peri Dial Int 2006; 26:178-82

33. Twardowski ZJ, Prowant BF. Current approach to exit-site infections in patients on peritoneal dialysis. Nephrol Dial Transplant 1997; 12:1284-95.

34. Fukasawa M, Matsushita K, Tanabe N, Mochizuki T, Hara T, Takeda M. A novel salvage technique that does not require catheter removal for exit-site infection. Perit Dial Int 2002; 22: 618-21.

35. Cheung AH, Wheeler MS, Limm WM, Wong LL, Pang RK, Chinn HY, Wong LM. A salvage technique for continuous ambulatory peritoneal dialysis catheters with exit-site infections. Am J Surg 1995; 170:60-1.

36. Crabtree JH. Peritoneal catheter splicing for distant relocation of poorly selected exit sites. Perit Dial Int 2005; 25:192-5.

37. Crabtree JH, Burchette RJ. Surgical salvage of peritoneal dialysis catheters from chronic exit-site and tunnel infections. The American Journal of Surgery 2005; 190:4-8.

38. Terawaki H, Nakano H, Ogura M, Kadomura M, Hosoya T, Nakayama M. Unroofing surgery with en bloc resection of skin and tissues around the peripheral cuff. Perit Dial Int 2013; 33:573-9.

39. Kirmizis D, Bowes E, Behzad A, Cairns H. Exit-site relocation: a novel, straightforward technique for exit-site infectious. Perit Dial Int 2019; 39:350-5.

40. Mari G, Scanziani R, Auricchio S, Crippa J, Maggioni D. Laparoscopic surgery in patients on peritoneal dialysis: a review of the literature. Surgical Innovation 2017; 24(4):397-401.

41. Goel S, Ribby KJ, Kathuria P, Khanna R. Temporary stoppage of peritoneal dialysis when laparoscopic procedures are performed on patients undergoing CAPD/CCPD: a change in policy. Adv Perito Dial. 1998; 14:80-2.

42. Moffat FL, Deitel M, Thompson DA. Abdominal surgery in patients undergoing long-term peritoneal dialysis. Surgery 2 1982:598-604.

43. Magnuson TH, Bender JS, Campbell KA, Ratner LE. Cholecystectomy in the peritoneal dialysis patient: unique advantages to the laparoscopic approach. Surg Endosc 1995;9(8):908-9.

44. Janez J. Synchronous Laparoscopic insertion of peritoneal dialysis catheter and cholecystectomy in patients with end-stage renal disease and gallstones - our experience. Perit Dial Int 2019; 39(5):489-91.

\section{ORCID}

Rita Vicente iD 0000-0003-2726-9539

Rui Silva iD 0000-0001-9933-9271

Ricardo Santos (iD) 0000-0003-4211-9942

Anabela Rodrigues (iD) 0000-0001-8818-2141

Manuel Amoedo (D) 0000-0003-3105-1559

\section{Correspondence to:}

Rita Vicente, MD

Nephrology Department, Hospital Espírito Santo de Évora, Portugal 